\title{
A LIFETIME OF EXCURSIONS THROUGH RANDOM WALKS AND LÉVY PROCESSES
}

\author{
LOÏC CHAUMONT AND ANDREAS KYPRIANOU
}

\begin{abstract}
In celebration of Professor Ron Doney's 80th birthday, we provide a summary of his academic career and contributions to probability theory, as one of the UK's leading probabilists for over 50 years. A version of this note also serves as an introductory article to the volume in honor of Ron Doney [Birkhäuser, 2021], which includes an additional 17 research papers produced by Ron's colleagues and friends.
\end{abstract}

Through this volume, it is with the greatest of admiration that we pay tribute to the mathematical achievements of Professor Ron Doney. His career has spanned generations of probabilists and his work continues to play a significant role in the community. In addition to the major contributions he has made in the theory of random walks and Lévy processes, Ron is equally appreciated for the support he has given to younger colleagues. The sentiment and desire to organise both a workshop and this volume to honour his lifetime achievements surfaced naturally a couple of years ago at the Lévy process meeting in Samos, as it became apparent that Ron was approaching his 80th birthday. A huge appreciation of his standing in the community meant it was very easy to find willing participation in both projects. As a prelude to this hommage à Ron, let us spend a little time reflecting on his career and his main achievements.

Ron grew up in working-class Salford in the North West of Greater Manchester at a time when few leaving school would attend university. Having a veracious appetite for reading, Ron spent long hours as a schoolboy in Manchester Central Library where he cultivated his intellect. Coupled with an obvious talent for mathematics, he found his way to the University of Durham. There he studied Mathematics as an undergraduate and continued all the way to a PhD under the supervision of Harry Reuter, graduating in 1964 aged just 24. Ron's thesis entitled 'Some problems on random walks' was no doubt inspired by the shift in interests of Harry Reuter from analysis to probability. At the time of his $\mathrm{PhD}$, all doctoral activity in probability theory in the UK was essentially supervised either by Harry Reuter or David Kendall. Because of this, Reuter and Kendall formed StAG, the Stochastic Analysis Group, which would meet regularly under the auspices of the London Mathematical Society. From a very young age, Ron thus had the opportunity to engage with his contemporaries. Back in the 1960s they were precious few in number compared with vast numbers of the probability PhD community present today in the UK and included the likes of Rollo Davidson, David Williams, Daryl Daley, David Vere-Jones, Nick Bingham, John Hawkes and John Kingman.

The time that Ron completed his $\mathrm{PhD}$ coincided with a period of expansion in the UK higher education system which proved to offer numerous opportunities during the first years of his academic career. From his PhD, Ron was successfully appointed directly to a lectureship at the University of East Anglia. He spent the academic year 1964-65 there, but quickly moved on to lectureship at Imperial College London in 1965, coinciding with the appointment of Harry Reuter to a chair there. During this early phase, Ron had a slow start to his publication record. His first two papers [1, 3] concern random walks 
in three dimensions, followed by a paper concerning higher dimensional version of the renewal theory [2].

By 1970, Ron had moved back to his native Manchester. He joined the then worldfamous Manchester-Sheffield School of Probability and Statistics, formally taking up a lectureship at Manchester's Statistical Laboratory. At this point in time he moved away from his initial work on random walks to the theory of Galton-Watson processes and, what were then called, general branching processes (today they would rather come under the heading of Crump-Mode-Jagers (CMJ) processes). Although seemingly a change in direction, this was a very natural move for anyone who harboured interests in random walks and renewal processes. Indeed, whilst the theory of branching processes has become significantly more exotic in recent years, the interplay of these two fields still remains highly pertinent today. Concurrently with the work of Peter Jagers, Ron produced a cluster of articles through to the mid 1970s looking at growth properties of CMJ processes in which, among other things, he demonstrated the central role that renewal processes play; 4, 6, 5, 7, 8, 9, 11, 10, 12. As early as 1972, one finds computations in his work which echo what would later become known as the method of spines; 5. Here, Ron also made contributions to underlying functional equations and the so-called $x \log x$ condition, which precede a number of similar results in the setting of more general spatial models such as branching random walks. The papers, [8, 9] with Nick Bingham, are also interesting to reflect upon in terms of how collaborations of the day were conducted. In a period of no internet or email, and with the probability community in the UK being very few in number and thinly spread, Ron maintained communication with Nick through hand-written and mailed letters during the epoch of their overlapping interests. In a process that has largely been replaced by Google, this involved sharing sample calculations, summaries of articles they had found and broader mathematical ideas. In the case of Ron and Nick, this led not only two these two papers, but calculations that lay dormant, surfacing over a decade later in [23].

By 1977, Ron was back to random walks, albeit now in one dimension. As a passive observer, it appears that there was an awakening in Ron's understanding of how many problems still remained open for general one-dimensional random walks, particularly when looking at them in terms of excursions. Aside from some works on Markov chains and Brownian motion, [19, 18, 27], Ron's work focused mostly with random walks through the 1980s. The prominence of his contributions lay with his use of Spitzer's condition as well as the problem of characterising the moments and tail behaviour of ladder variables in relation to assumptions on the underlying random walk; [13, 14, 15, 16, 17, 26, 24, 25]. He began a growing interest in arcsine laws and random walks in the domain of attraction of stable laws as

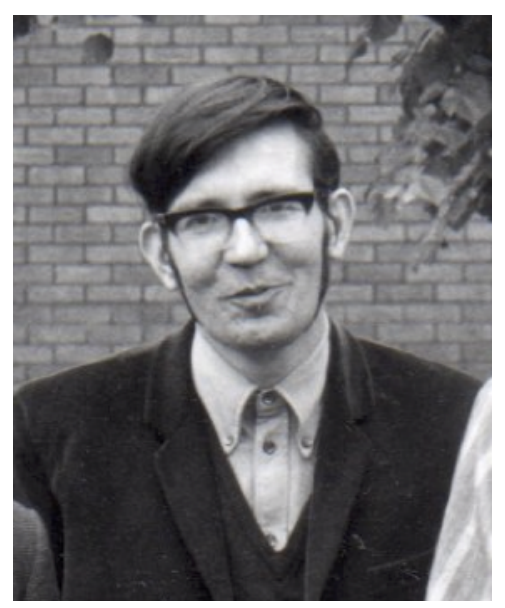

Ron Doney in 1972, Manchester. well as stable processes themselves; [20, 21, 22, 23]. Most notable of the latter is a remarkable paper on the Wiener-Hopf factorisation of stable processes [22, the significance of which would become apparent many years later after 2010 thanks to continued work of a number of 
authors, most notably Alexey Kuznetsov. It was also during that 1980s that Ron took two sabbaticals to Canada. The first stay was in Vancouver in 1980-81, visiting Cindy Greenwood, which also allowed him the chance to connect with Sidney Port, Ed Perkins and John Walsh. For the second, he was visiting George O'Brien in York University, Toronto in 1988-89.

Moving into the 1990s, many things changed for Ron's research, least of all, his rate of publication, 228, 30, 29, 31, 32, 35, 33, 34, 39, 36, 38, 37, 40, 41, 42, 44, 78, 48, 45, 47, 46, 49, The previous work he had done on ladder heights and Spitzer's condition culminated in one of Ron's most important and widely appreciated results: For random walks (and shortly after for Lévy processes), he proved that Spitzer's condition was equivalent to the convergence of the positivity probability, 40, 44. Ron found himself catapulted into a rapidly growing and much better organised global community of probabilists with shared interests to his own. Although he largely continued publishing in the context of random walks, it was during this decade that Ron became increasingly exposed to the theory of Lévy processes.

The 1990s was also the decade that saw Ron begin to jointly co-author many more of his papers. There were a number of factors at play here. During the first twenty five years of Ron's career it was quite common for academics to author papers alone, least of all because, within the field of probability theory, probabilists were few and far between. The 1990s was the decade of globalisation and the prevalence of email and internet made international connectivity and communication much easier. But perhaps most importantly in this respect was Ron's collaboration with a young French mathematician by the name of Jean Bertoin, which dramatically opened up his relationship with the Parisian (and more generally the French) school of probability for the rest of his career.

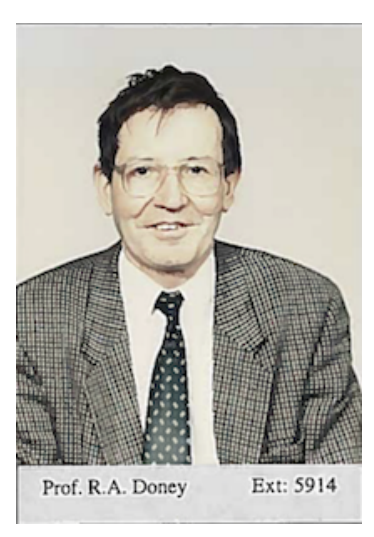

Ron Doney's departmental photo as it appeared on the entrance to the Mathematics Tower in the 1990 s.

In the early 1990s, Ron had made contact with Jean Bertoin, who was very interested in random walks, but also in capturing and expanding on the less-well explored theory of Lévy processes. It was Ron's first paper on Lévy processes, [29], which had caught the interest of Jean and they began sending each other preprints. They first met in person in Luminy during one of the Journées de Probabilités in 1992 organised by Azéma and Yor. At the time, Ron was interested in a paper by Keener on the simple RW conditioned to stay positive that had been published that year. Although others had written on the topic of conditioned random walks, it was the joint work of Jean and Ron [37] that ensued, which formalised a robust approach to the notion of such conditionings and led the way for a number of articles on conditioned processes, particularly in the setting of Lévy processes. Reflecting on earlier remarks about how the theory of random walks and branching processes are so closely intertwined, it is worth noting that the paper [37. ended up playing a hugely influential role in the theory of branching random walks, branching Brownian motion and (growth) fragmentation processes, where conditioning 
of the spine in the spirit of their work proved to be instrumental in understanding the so-called derivative martingale.

The collaboration between Jean and Ron was relatively intensive for a period of 3-4 years with six of their seven co-authored papers, [39, 36, 38, 37, 41, 43, 78, appearing between 1994 and 1997. During this period, Jean would visit Ron regularly, often working at his home in the village of Whaley Bridge, South East of Manchester. Their daily working routine would be interlaced with regular hikes out into the Peak District which lay just beyond Ron's back door.

Through his collaboration with Jean Bertoin, Ron also started to visit Paris more often. There, he became acquainted with a new generation of young probabilists who were being guided towards the theory of Lévy processes. Loïc Chaumont was the first PhD student of Jean who also became a long-time collaborator of Ron. Their collaboration spans 8 papers to date, [50, 51, 52, 66, 67, 79, 83, 98, in which they cover the study of perturbed Brownian motion, distributional decompositions of the general Wiener-Hopf factorisation and Lévy processes conditioned to stay positive. It is perhaps the latter, [67, 79, for which they are best known as co-authors, building on the $\mathrm{PhD}$ thesis of Loïc for conditioned Lévy processes that had, in turn, grown out of the formalisation for conditioned random walks that Ron had undertaken with Jean Bertoin. It was also during the mid 1990s that Larbi Alili, a contemporary of Loïc and PhD student of Marc Yor, became the postdoc of Ron on a competitively funded EPSRC project; cf [50, 49, 54]. Philippe Marchal, another gifted young probabilist from the Paris school, was also a regular visitor to Manchester during this period. Another young French probabilist whose work greatly impressed Ron at the turn of the Millennium was Vincent Vigon. Aside from Ron's admiration of Vincent's unexpected emergence from Rouen rather than Paris, what impressed him the most was that Vigon had established a necessary and sufficient condition, in the form of an explicit integral test, for when a Lévy process of unbounded variation with no Gaussian component creeps; in particular, this result showed that Ron's previous conjecture on this matter, which had been assimilated from the long-term behaviour of random walks, was wrong.

The new Millennium brought about yet further change for Ron. His publication rate went up yet another gear, with almost as many articles published during this decade as in the previous two. This was all thanks to his increased exposure to collaborative partnership as well as the inevitable depth of understanding of random walks and Lévy processes he had acquired; [51, 52, 55, 54, 56, 57, 53, 59, 60, 61, 62, 58, 63, 65, 64, 66, 72, 69, 68, 70, 67, 71, 74, 73, 76, 775, 77, 78, 79, 80, 82, 81. It was also during this decade that Ron began an extremely fruitful collaboration with Ross Maller, publishing 10 papers together; [53, 59, 58, 65, 70, 71, 76, 77, 78, 82, 94. On a visit to the UK, Ross was advised by Charles Goldie to go and spend time in Manchester visiting Ron. He did and they immediately started producing material. In a number of important papers [58, 59, 82], Ross and Ron first investigated the asymptotic behaviour of random walks and Lévy processes at deterministic times and at first passage times across a fixed level. In another series of remarkable works [71, 76, 77, 78] they considered first passage times across power law boundaries of random walks, Lévy processes and their reflected version at the infimum. In particular they obtained necessary and sufficient conditions for these first passage times to have finite moments. 
By now, there was widespread renewed interest in the theory of fluctuations for Lévy processes and the study of their overshoots had become very popular. Two articles by Ron, written with Phil Griffin [60], 64] bear witness to this. It was also during this time that Ron collaborated with Andreas Kyprianou and wrote one of his most cited articles on the so-called quintuple law. The latter gives a distributional identity for a suite of five important and commonly used path functionals of a Lévy process at first passage over a fixed level in terms of its ladder potentials and Lévy measure. In essence, the result constitutes a 'disintegration' of the Wiener-Hopf factorisation. Ron also began a fruitful collaboration with Mladen Savov during this period; [82, 85, 84]. Mladen, who came to Manchester from Buglaria, proved to be the most accomplished of Ron's several PhD students.

A very important event of this decade for Ron was his invitation to give a lecture at the famous Saint-Flour summer school in 2005. It was arguably the first major recognition of Ron's career by the mathematical community. For Ron, this also presented the opportunity to write a book on fluctuation theory for Lévy processes, [75], which remains an important reference in the domain to date. Ron found himself centre-stage as part of a huge community of researchers now working specifically in the field of random walks and Lévy processes. He spoke at many venues, including a rapid succession of workshops and congresses devoted to Lévy processes and was elected as a Fellow of the Institute of Mathematical Statistics in 2006. Moreover, in 2005, Manchester hosted the 4th international workshop on Lévy processes. This was a huge undertaking given the large number of attendees, but nonetheless an important moment that asserted the importance of Manchester's probability group and, in particular, Ron's identity as a highly accomplished researcher in this field.

The next decade, 2010-2020, saw an invitation by the Bernoulli Society for Ron to give a prestigious plenary lecture at the 2013 Stochastic Processes and their Applications congress in Boulder, Colorado. Together with his lectures at Saint Flour, this stands as quite an important recognition of his achievements from the international mathematical community. Technically speaking, Ron retired in 2006, however, as a special case given the quality of his research output, Manchester had gladly continued his appointment beyond his 67th birthday, right through until 2014, on a purely research basis. Now in his 70s, one might say Ron worked at a slower rate, however, although publishing in lower vol-

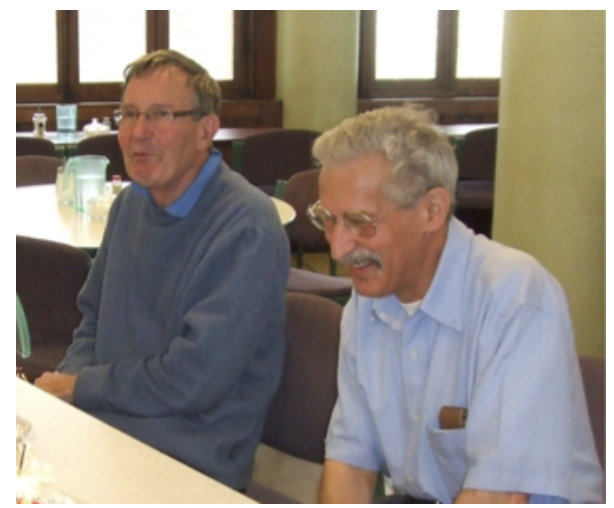

Taken on the 24th July 2009 at the 8th International Conference on Lévy processes, held in Angers, Ron Doney sits with Marc Yor to celebrate Marc's 60th birthday. ume, one sees remarkable crowning quality in his work with an exceptional number of his papers appearing in the top two probability journals Annals of Probability and Probability Theory and Related Fields; [85, 83, 84, 86, 89, 88, 87, 90, 91, 92, 93, 94, 95, 96, 97, 99, 98]. 
These works are marked by two projects in which Ron revisited old obsessions. The first is a cluster of papers, co-authored with Víctor Rivero. Ron went back to the first passage times of Lévy processes and obtained sharp results for the local behaviour of their distribution, [91], [93], [95]. The second concerns improving the classical Gnedenko and Stone local limit theorems. In collaboration with Francesco Caravenna [97], Ron obtained necessary and sufficient conditions for random walks in the domain of attraction of a stable law to satisfy the strong renewal theorem. This work, as well as [99, solve a long-standing problem which dates back to the 1960's. This second achievement is the one that Ron himself quietly admits he is most proud of, and rightly so.

As Ron's career wound down, he had the pleasure of watching the probability group in Manchester dramatically grow in size, with 10 members around the time of his final retirement, something he had dreamed of for many years. Ron was appointed in Manchester at a time that it stood as a global stronghold for probability and statistics. The Manchester Statistical Laboratory was half of the very unique two-university ManchesterSheffield School of Probability and Statistics, the brain child of Joe Gani. With changing times the Manchester-Sheffield School disbanded and, aside from Fredos Papangelou, who joined in 1973, Ron was the only other probabilist who remained in the Statistical Laboratory for a number of years to come. The 1980s were hard times for British academia, but the growth in the international community around Ron's research interests through the 1990s was mirrored in the growth of the probability group in Manchester. The turn of the Millennium saw the merger of the University of Manchester (or rather, more formally, the Victoria University of Manchester) with UMIST (University of Manchester Institute of Science and Technology), which opened the door to new opportunities. Many of the probability appointments in Manchester since then clearly reflect the strong association of Manchester with the theory of random walks and Lévy processes; something that is directly tethered to Ron's towering achievements as a researcher.

As alluded to at the start of this article, Ron is appreciated as much for his encouragement of young researchers as he is for the mathematics that he has produced. Just as the authors of this article see Ron as one of the major influencing characters in their careers, both through academic mentorship and mathematical discourse, so do many others among our community, both in the UK and around the globe. There are simply too many to list here, moreover, an attempt to do so would carry the risk that we forget names. But it should be said that, when the idea of holding a workshop for Ron's 80th birthday surfaced, this was carried forward by an emotional surge of support from the many who belong to the aforementioned list.

As a humble researcher who cares little for the limelight, Ron did not always get the honours he deserved. Towards his retirement, the number of researchers in probability theory exploded exponentially and Ron's contribution to a classical field, which now lies in the DNA of many modern research endeavours, is often overlooked. One goal of this volume and the accompanying conference is to try to correct this.

We hope the contents of this volume will stimulate Ron to think about his next piece of work. He currently has 99 publications and we are all keenly awaiting his 100th! 


\section{REFERENCES}

[1] Doney, R. A. Recurrent and transient sets for 3-dimensional random walks. Z. Wahrscheinlichkeitstheorie und Verw. Gebiete, 4, (1965), 253-259.

[2] Doney, R. A. An analogue of the renewal theorem in higher dimensions. Proc. London Math. Soc. (3), 16, (1966), 669-684.

[3] Doney, R. A. Hits on an axis by the simple random walk in three dimensions. Z. Wahrscheinlichkeitstheorie und Verw. Gebiete, 5, (1966), 146-155.

[4] Doney, R. A. The progeny of a branching process. J. Appl. Probability, 8, (1971), 407-412.

[5] Doney, R. A. A limit theorem for a class of supercritical branching processes. J. Appl. Probability, 9, (1972), 707-724.

[6] R. A. Doney. Age-dependent birth and death processes. Z. Wahrscheinlichkeitstheorie und Verw. Gebiete 22 (1972), pp. 69-90.

[7] R. A. Doney. On a functional equation for general branching processes. J. Appl. Probability 10 (1973), pp. $198-205$.

[8] N. H. Bingham and R. A. Doney. Asymptotic properties of supercritical branching processes. I. The Galton-Watson process. Advances in Appl. Probability 6 (1974), pp. 711-731.

[9] N. H. Bingham and R. A. Doney. Asymptotic properties of supercritical branching processes. II. Crump-Mode and Jirina processes. Advances in Appl. Probability 7 (1975), pp. 66-82.

[10] R. A. Doney. A note on the subcritical generalized age-dependent branching pro- cess. J. Appl. Probability 13.4 (1976), pp. 798-803.

[11] R. A. Doney. On single- and multi-type general age-dependent branching processes. J. Appl. Probability 13.2 (1976), pp. 239-246.

[12] R. A. Doney. A note on a condition satisfied by certain random walks. J. Appl. Probability 14.4 (1977), pp. 843-849.

[13] R. A. Doney. Moments of ladder heights in random walks. J. Appl. Probab. 17.1 (1980), pp. 248-252.

[14] R. A. Doney. Spitzer's condition for asymptotically symmetric random walk. J. Appl. Probab. 17.3 (1980), pp. 856-859.

[15] R. A. Doney. On the exact asymptotic behaviour of the distribution of ladder epochs. Stochastic Process. Appl. 12.2 (1982), pp. 203-214.

[16] R. A. Doney. On the existence of the mean ladder height for random walk. Z. Wahrsch. Verw. Gebiete 59.3 (1982), pp. 373-382.

[17] R. A. Doney. A note on conditioned random walk. J. Appl. Probab. 20.2 (1983), pp. 409-412.

[18] R. A. Doney. A note on some results of Schuh. J. Appl. Probab. 21.1 (1984), pp. 192-196.

[19] R. A. Doney. Letter to the editor: On conditional passage time structure of birth- death processes [J. Appl. Probab. 21 (1984), no. 1, 10-21] by U. Sumita. J. Appl. Probab. 21.3 (1984), pp. 673-674.

[20] R. A. Doney. Conditional limit theorems for asymptotically stable random walks. Z. Wahrsch. Verw. Gebiete 70.3 (1985), pp. 351-360.

[21] R. A. Doney. On the maxima of random walks and stable processes and the arc-sine law. Bull. London Math. Soc. 19.2 (1987), pp. 177-182.

[22] R. A. Doney. On Wiener-Hopf factorisation and the distribution of extrema for certain stable processes. Ann. Probab. 15.4 (1987), pp. 1352-1362.

[23] N. H. Bingham and R. A. Doney. On higher-dimensional analogues of the arc-sine law. J. Appl. Probab. 25.1 (1988), pp. 120-131.

[24] R. A. Doney. A large deviation local limit theorem. Math. Proc. Cambridge Philos. Soc. 105.3 (1989), pp. $575-577$.

[25] R. A. Doney. Last exit times for random walks. Stochastic Process. Appl. 31.2 (1989), pp. 321-331.

[26] R. A. Doney. On the asymptotic behaviour of first passage times for transient random walk. Probab. Theory Related Fields 81.2 (1989), pp. 239-246.

[27] R. A. Doney and D. R. Grey. Some remarks on Brownian motion with drift. J. Appl. Probab. 26.3 (1989), pp. 659-663.

[28] R. A. Doney. A bivariate local limit theorem. J. Multivariate Anal. 36.1 (1991), pp. 95-102.

[29] R. A. Doney. Hitting probabilities for spectrally positive Lévy processes". J. London Math. Soc. (2) 44.3 (1991), pp. 566-576.

[30] R. A. Doney and G. L. O'Brien. Loud shot noise. Ann. Appl. Probab. 1.1 (1991), pp. 88-103. 
[31] R. A. Doney and C. M. Goldie. Letter to the editor: Autoregressive processes and first-hit probabilities for randomized random walks [J. Appl. Probab. 28 (1991), no. 2, 347-359] by R. Epstein-Feldman. J. Appl. Probab. 29.1 (1992), p. 244.

[32] R. A. Doney and G. L. O'Brien. Thickened renewal processes. Stochastic Process. Appl. 43.1 (1992), pp. $1-8$.

[33] R. A. Doney. A path decomposition for Lévy processes. Stochastic Process. Appl. 47.2 (1993), pp. 167-181.

[34] R. A. Doney. Some results involving the maximum of Brownian motion. J. Appl. Probab. 30.4 (1993), pp. $805-818$.

[35] R. A. Doney and P. E. Greenwood. On the joint distribution of ladder variables of random walk. Probab. Theory Related Fields 94.4 (1993), pp. 457-472.

[36] J. Bertoin and R. A. Doney. Cramér's estimate for Lévy processes. Statist. Probab. Lett. 21.5 (1994), pp. 363-365.

[37] J. Bertoin and R. A. Doney. On conditioning a random walk to stay nonnegative. Ann. Probab. 22.4 (1994), pp. 2152-2167.

[38] J. Bertoin and R. A. Doney. On conditioning random walks in an exponential family to stay nonnegative. Séminaire de Probabilités, XXVIII. Vol. 1583. Lecture Notes in Math. Springer, Berlin, 1994, pp. 116-121.

[39] J. Bertoin and R. A. Doney. On the local behaviour of ladder height distributions. J. Appl. Probab. 31.3 (1994), pp. 816-821.

[40] R. A. Doney. Spitzer's condition and ladder variables in random walks. Probab. Theory Related Fields 101.4 (1995), pp. 577-580.

[41] J. Bertoin and R. A. Doney. Some asymptotic results for transient random walks. Adv. in Appl. Probab. 28.1 (1996), pp. 207-226.

[42] R. A. Doney. Increase of Lévy processes. Ann. Probab. 24.2 (1996), pp. 961-970.

[43] J. Bertoin and R. A. Doney. Spitzer's condition for random walks and Lévy processes. Ann. Inst. H. Poincaré Probab. Statist. 33.2 (1997), pp. 167-178.

[44] R. A. Doney. One-sided local large deviation and renewal theorems in the case of infinite mean. Probab. Theory Related Fields 107.4 (1997), pp. 451-465.

[45] R. A. Doney. Some calculations for perturbed Brownian motion. Séminaire de Probabilités, XXXII. Vol. 1686. Lecture Notes in Math. Springer, Berlin, 1998, pp. 231-236.

[46] R. A. Doney. The Martin boundary and ratio limit theorems for killed random walks. J. London Math. Soc. (2) 58.3 (1998), pp. 761-768.

[47] R. A. Doney, J. Warren, and M. Yor. Perturbed Bessel processes. Séminaire de Probabilités, XXXII. Vol. 1686. Lecture Notes in Math. Springer, Berlin, 1998, pp. 237-249.

[48] R. A. Doney and M. Yor. On a formula of Takács for Brownian motion with drift. J. Appl. Probab. 35.2 (1998), pp. 272-280.

[49] L. Alili and R. A. Doney. Wiener-Hopf factorization revisited and some applications. Stochastics Stochastics Rep. 66.1-2 (1999), pp. 87-102.

[50] L. Chaumont and R. A. Doney. Pathwise uniqueness for perturbed versions of Brownian motion and reflected Brownian motion. Probab. Theory Related Fields 113.4 (1999), pp. 519-534.

[51] L. Chaumont and R. A. Doney. Some calculations for doubly perturbed Brownian motion. Stochastic Process. Appl. 85.1 (2000), pp. 61-74.

[52] L. Chaumont, R. A. Doney, and Y. Hu. Upper and lower limits of doubly perturbed Brownian motion. Ann. Inst. H. Poincaré Probab. Statist. 36.2 (2000), pp. 219-249.

[53] R. A. Doney and R. A. Maller. Random walks crossing curved boundaries: a functional limit theorem, stability and asymptotic distributions for exit times and positions. Adv. in Appl. Probab. 32.4 (2000), pp. 1117-1149.

[54] L. Alili and R. A. Doney. Martin boundaries associated with a killed random walk. Ann. Inst. H. Poincaré Probab. Statist. 37.3 (2001), pp. 313-338.

[55] R. A. Doney. A local limit theorem for moderate deviations. Bull. London Math. Soc. 33.1 (2001), pp. 100-108.

[56] R. A. Doney. Fluctuation theory for Lévy processes. Birkhäuser Boston, Boston, MA, 2001, pp. 57-66.

[57] R. A. Doney and Y. B. Nakhi. Perturbed and non-perturbed Brownian taboo processes. Ann. Inst. H. Poincaré Probab. Statist. 37.6 (2001), pp. 725-736.

[58] R. A. Doney and R. A. Maller. Stability and attraction to normality for Lévy processes at zero and at infinity. J. Theoret. Probab. 15.3 (2002), pp. 751-792. 
[59] R. A. Doney and R. A. Maller. Stability of the overshoot for Lévy processes. Ann. Probab. 30.1 (2002), pp. 188-212.

[60] R. A. Doney and P. S. Griffin. Overshoots over curved boundaries. Adv. in Appl. Probab. 35.2 (2003), pp. 417-448.

[61] R. A. Doney and P. Marchal. A third arc-sine theorem. Bull. London Math. Soc. 35.4 (2003), pp. $536-540$.

[62] R. A. Doney. Small-time behaviour of Lévy processes. Electron. J. Probab. 9 (2004), no. 8, $209-229$.

[63] R. A. Doney. Stochastic bounds for Lévy processes. Ann. Probab. 32.2 (2004), pp. 1545-1552.

[64] R. A. Doney and P. S. Griffin. Overshoots over curved boundaries. II. Adv. in Appl. Probab. 36.4 (2004), pp. 1148-1174.

[65] R. A. Doney and R. A. Maller. Moments of passage times for Lévy processes. Ann. Inst. H. Poincaré Probab. Statist. 40.3 (2004), pp. 279-297.

[66] L. Alili, L. Chaumont, and R. A. Doney. On a fluctuation identity for random walks and Lévy processes. Bull. London Math. Soc. 37.1 (2005), pp. 141-148.

[67] L. Chaumont and R. A. Doney. On Lévy processes conditioned to stay positive. Electron. J. Probab. 10 (2005), no. 28, 948-961.

[68] R. A. Doney. Some excursion calculations for spectrally one-sided Lévy processes. Séminaire de Probabilités XXXVIII. Vol. 1857. Lecture Notes in Math. Springer, Berlin, 2005, pp. 5-15.

[69] R. A. Doney. Tanaka's construction for random walks and Lévy processes. Séminaire de Probabilités XXXVIII. Vol. 1857. Lecture Notes in Math. Springer, Berlin, 2005, pp. 1-4.

[70] R. A. Doney and R. A. Maller. Cramér's estimate for a reflected Lévy process. Ann. Appl. Probab. 15.2 (2005), pp. 1445-1450.

[71] R. A. Doney and R. A. Maller. Passage times of random walks and Lévy processes across power law boundaries. Probab. Theory Related Fields 133.1 (2005), pp. 57-70.

[72] R. A. Doney and T. Zhang. Perturbed Skorohod equations and perturbed reflected diffusion processes. Ann. Inst. H. Poincaré Probab. Statist. 41.1 (2005), pp. 107-121.

[73] A. Bryn-Jones and R. A. Doney. A functional limit theorem for random walk conditioned to stay non-negative. J. London Math. Soc. (2) 74.1 (2006), pp. 244-258.

[74] R. A. Doney and A. E. Kyprianou. Overshoots and undershoots of Lévy processes. Ann. Appl. Probab. 16.1 (2006), pp. 91-106.

[75] R. A. Doney. Fluctuation theory for Lévy processes. Vol. 1897. Lecture Notes in Mathematics. Lectures from the 35th Summer School on Probability Theory held in Saint-Flour, July 6-23, 2005, Edited and with a foreword by Jean Picard. Springer, Berlin, 2007, pp. $\mathrm{x}+147$.

[76] R. A. Doney and R. A. Maller. Almost sure relative stability of the overshoot of power law boundaries. J. Theoret. Probab. 20.1 (2007), pp. 47-63.

[77] R. A. Doney and R. A. Maller. Curve crossing for random walks reflected at their maximum. Ann. Probab. 35.4 (2007), pp. 1351-1373.

[78] J. Bertoin, R. A. Doney, and R. A. Maller. Passage of Lévy processes across power law boundaries at small times. Ann. Probab. 36.1 (2008), pp. 160-197.

[79] L. Chaumont and R. A. Doney. Corrections to: On Lévy processes conditioned to stay positive [Electron J. Probab. 10 (2005), no. 28, 948-961]. Electron. J. Probab. 13 (2008), no. 1, 1-4.

[80] R. A. Doney. A note on the supremum of a stable process. Stochastics 80.2-3, IMS Lecture NotesMonograph Series (2008), pp. 151-155.

[81] R. A. Doney. Asymptotic analysis of random walks: heavy-tailed distributions. J. Amer. Statist. Assoc. 104.488 (2009), p. 1714.

[82] R. A. Doney, R. A. Maller, and M. S. Savov. Renewal theorems and stability for the reflected process. Stochastic Process. Appl. 119.4 (2009), pp. 1270-1297.

[83] L. Chaumont and R. A. Doney. Invariance principles for local times at the maximum of random walks and Lévy processes. Ann. Probab. 38.4 (2010), pp. 1368-1389.

[84] R. A. Doney and M. S. Savov. Right inverses of Lévy processes. Ann. Probab. 38.4 (2010), pp. 1390-1400.

[85] R. A. Doney and M. S. Savov. The asymptotic behavior of densities related to the supremum of a stable process. Ann. Probab. 38.1 (2010), pp. 316-326.

[86] R. A. Doney and D. A. Korshunov. Local asymptotics for the time of first return to the origin of transient random walk. Statist. Probab. Lett. 81.9 (2011), pp. 1419-1424.

[87] Bertoin, J. and Bretagnolle, J. L. and Doney, R. A. and Ibragimov, I. A. and Jacod, J. Lévy processes at Saint-Flour. Probability at Saint-Flour. Springer, Heidelberg, 2012, pp. viii +476 . 
[88] R. A. Doney. Local behaviour of first passage probabilities. Probab. Theory Related Fields 152.3-4 (2012), pp. 559-588.

[89] R. A. Doney and E. M. Jones. Conditioned random walks and Lévy processes. Bull. Lond. Math. Soc. 44.1 (2012), pp. 139-150.

[90] R. A. Doney and E. M. Jones. Large deviation results for random walks conditioned to stay positive. Electron. Commun. Probab. 17 (2012), no. 38, 11.

[91] R. A. Doney and V. Rivero. Asymptotic behaviour of first passage time distributions for Lévy processes. Probab. Theory Related Fields 157.1-2 (2013), pp. 1-45.

[92] R. A. Doney and S. Vakeroudis. Windings of planar stable processes. Séminaire de Probabilités XLV. Vol. 2078. Lecture Notes in Math. Springer, Cham, 2013, pp. 277-300.

[93] R. A. Doney and Víctor M. Rivero. Asymptotic behaviour of first passage time distributions for subordinators. Electron. J. Probab. 20 (2015), no. 91, 28.

[94] R. A. Doney, C. Klüppelberg, and R. A. Maller. Passage time and fluctuation calculations for subexponential Lévy processes. Bernoulli 22.3 (2016), pp. 1491-1519.

[95] R. A. Doney and V. Rivero. Erratum to: Asymptotic behaviour of first passage time distributions for Lévy processes. Probab. Theory Related Fields 164.3-4 (2016), pp. 1079-1083.

[96] R. A. Doney and P. S. Griffin. Cramér's estimate for the reflected process revisited. Ann. Appl. Probab. 28.6 (2018), pp. 3629-3651.

[97] Francesco Caravenna and R. A. Doney. Local large deviations and the strong renewal theorem. Electron. J. Probab. 24 (2019), Paper No. 72, 48.

[98] L. Chaumont and R. A. Doney. On distributions determined by their upward, space-time WienerHopf factor. J. Theoret. Probab. 33.2 (2020), pp. 1011-1033.

[99] R. A. Doney. The remainder in the renewal theorem. Electron. Commun. Probab. 25 (2020), Paper No. 5,8 .

LoḮ Chaumont, Univ Angers, CNRS, LAREMA, SFR MATHSTIC, F-49000 Angers, France

Email address: loic.chaumont@univ-angers.fr

Andreas Kyprianou - Department of Mathematical Sciences, University of Bath, ClaverTON DOWn, BA2 7AY

Email address: a.kyprianou@bath.ac.uk 